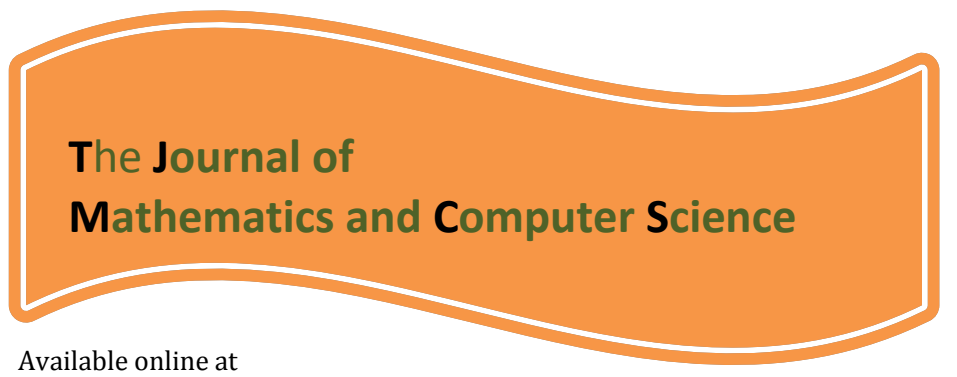

http://www.TJMCS.com

The Journal of Mathematics and Computer Science Vol. 4 No.3 (2012) 386 - 391

\title{
Existence of Three Weak Solutions for Elliptic Dirichlet Problem
}

\author{
G.A. Afroiuzi ${ }^{1 *}$, T.N. Ghara ${ }^{2}$ \\ ${ }^{1}$ Department of Mathematics, Faculty of Mathematical Sciences, \\ University of Mazandaran, Babolsar, Iran \\ afrouzi@umz.ac.ir \\ ${ }^{2}$ Department of Mathematics, Faculty of Mathematical Sciences, University of \\ Mazandaran, Babolsar, Iran \\ tahere.noruzi5@gmail.com
}

Received: February 2012, Revised: May 2012

Online Publication: July 2012

\begin{abstract}
The aim of this paper is to establish the existence of at least three weak solutions for the elliptic Dirichlet problem. Our main tool is a three critical point theorem and Therorem3.1. of Gabriele Bonanno, Giovanni Molica Bisci [4].
\end{abstract}

Keywords: Dirichlet problem; Critical points; Three noitulos

\section{Introduction}

In this paper we investigate the following elliptic Dirichlet problem

$$
\left\{\begin{array}{lr}
-\Delta u=\lambda f(x, u)-a(x) u & \text { in } \Omega \\
u=0 & \text { on } \partial \Omega
\end{array}\right.
$$

where $\Omega$ is a non empty bounded open subset of the Euclidean space $\left(R^{N},||.\right), \mathrm{N} \geq 3$, with boundary of class $C^{1}, \lambda$ is a positive parameter and $\mathrm{f}: \Omega \times R \rightarrow R$ is a function, and the positive weight function $\mathrm{a}(\mathrm{x}) \in C(\bar{\Omega})$.

Existence of three solutions for different kinds of Dirichlet problem has been widely studied in literature, see for instance[1, 3, 5, 6,7].

\section{Preliminaries}

Our main tool is the following critical point theorem.

\footnotetext{
${ }^{*}$ Corresponding author
} 
Theorem 2.1. Let $X$ be a reflexive real Banach space, $\phi: X \rightarrow R$ be a coercive, continuously Gâteaux differentiable and sequentially weakly lower semicontinuous functional whose Gâteaux derivative admits a continuous invers on $X^{*}, \psi: X \rightarrow R$ be a continuously Gâteaux differentiable functional whose Gâteaux derivative is compact such that $\phi(0)=\psi(0)=0$.

Assume that there exist $\mathrm{r}>0$ and $\bar{x} \in X$, with $\mathrm{r}<\phi(\bar{x})$, such that:

$\left(a_{1}\right) \frac{\sup _{\phi(x) \leq r} \psi(x)}{r}<\frac{\psi(\bar{x})}{\phi(\bar{x})}$;

$\left(a_{2}\right)$ hcae $\left.\operatorname{rof} \lambda \in \Lambda_{r}:=\right] \frac{\psi(\bar{x})}{\phi(\bar{x})}, \frac{r}{\sup _{\phi(x) \leq r} \psi(x)}[$ the functional $\phi-\lambda \psi$ is coercive.

Then, for each $\lambda \in \Lambda_{r}$, the functional $J_{\lambda}:=\phi-\lambda \psi$ has at least three distinct critical points in X. Here and in the sequal, $\mathrm{f}: \Omega \times R \rightarrow R$ is a Caratheodory function such that

$\left(h_{1}\right)$ There exist two non negative constants $a_{1}, a_{2}$ and $\left.\mathrm{q} \in\right] 1, \frac{2 N}{(N-2)}[$ such that

$$
|\mathrm{f}(\mathrm{x}, \mathrm{t})| \leq a_{1}+a_{2}|t|^{q-1},
$$

for every $(\mathrm{x}, \mathrm{t}) \in \Omega \times R$.

We recall that the symbol $H_{0}^{1}(\Omega)$ indicates the closure of $C_{0}^{\infty}(\Omega)$ in the Sobolev space $W^{1,2}(\Omega)$, with respect to the norm

and we define

$$
\|\mathrm{u}\|:=\left(\int_{\Omega}|\nabla u(x)|^{2} d x\right)^{\frac{1}{2}}
$$

$$
\left.|| u\right|_{I}:=\left(\int_{\Omega}\left(|\nabla u(x)|^{2}+a(x)|u(x)|^{2}\right) d x\right)^{\frac{1}{2}}
$$

then there exist positive suitable constants $c_{1}, c_{2}$ such that

$$
c_{1}\|u\| \leq\|u\|\left\|_{I} \leq c_{2}\right\| u \|
$$

and a function $\mathrm{u}: \Omega \rightarrow R$ is said to be a weak solution of (1.1) if $\mathrm{u} \in H_{0}^{1}(\Omega)$ and

for all $v \in H_{0}^{1}(\Omega)$.

$$
\int_{\Omega} \nabla u(x) \nabla v(x) d x-\lambda \int_{\Omega} f(x, u(x)) v(x) d x=-\int_{\Omega} a(x) u(x) v(x) d x
$$

In order to study problem (1.1), we will use the functionals $\phi, \psi: H_{0}^{1}(\Omega) \rightarrow R$ defined by putting

and

$$
\phi(u):=\frac{\|u\|_{I}^{2}}{2},
$$

Where

$$
\psi(u):=\int_{\Omega} F(x, u(x)) d x, \quad \forall u \in H_{0}^{1}(\Omega),
$$

for every $(\mathrm{x}, \xi) \in \Omega \times R$.

$$
\mathrm{F}(\mathrm{x}, \xi):=\int_{0}^{\xi} f(x, t) d t
$$

From [4] clearly $\phi: X \rightarrow R$ is a coercive, continuously Gâteaux differentiable and sequentially weakly lower semi continuous functional whose Gâteaux derivative admits a continuous inverse on $X^{*}$. On the other hand, $\psi$ is well defined, continuously Gâteaux differentiable and with compact derivative. More precisely, one has

$$
\begin{gathered}
\dot{\phi}(u)(v)=\int_{\Omega}(\nabla u(x) \cdot \nabla v(x)+a(x) u(x) v(x)) d x, \\
\dot{\psi}(u)(v)=\int_{\Omega} f(x, u(x)) v(x) d x,
\end{gathered}
$$


for every $\mathrm{u}, \mathrm{v} \in H_{0}^{1}(\Omega)$.

A critical point of the functional $J_{\lambda}:=\phi-\lambda \psi$ is a function $u \in H_{0}^{1}(\Omega)$ such that

$$
\hat{\phi}(u)(v)-\lambda \dot{\psi}(u)(v)=0,
$$

for every $\mathrm{v} \in H_{0}^{1}(\Omega)$. Hence the critical points of the functional $J_{\lambda}$ are weak solutions of problem (1.1). Now, put $2^{*}=\frac{2 N}{(N-2)}$ and denote, as usual, with $\Gamma$ the Gamma function defined by

$$
\Gamma(t):=\int_{0}^{+\infty} z^{t-1} e^{-z} d z, \quad \forall t>0 .
$$

From the Sobolev embedding theorem there exist $c \in R^{+}$such that

$$
\|u\|_{L^{2^{*}}(\Omega)} \leq c|| u \|, \quad u \in H_{0}^{1}(\Omega) .
$$

The best constant that appears in (2.3) is

$$
\mathrm{c}=\frac{1}{\sqrt{N(N-2) \pi}}\left(\frac{N !}{2 \Gamma\left(1+\frac{N}{2}\right)}\right)^{\frac{1}{N}},
$$

Fixing $\mathrm{q} \in\left[1,2^{*}\left[\right.\right.$, again from the Sobolev embedding theorem, there exists a positive constant $c_{q}$ such that

$$
\|u\|_{L^{q}(\Omega)} \leq c_{q}|| u \|, \quad u \in H_{0}^{1}(\Omega)
$$

and, in particular, the embedding $H_{0}^{1}(\Omega) \hookrightarrow L^{q}(\Omega)$ is compact.

Due to (2.4), as simple consequence of Holder's inequality, it follows that

$$
c_{q} \leq \frac{\text { meas }(\Omega)^{\frac{2^{*}-q}{2^{*} q}}}{\sqrt{N(N-2) \pi}}\left(\frac{N !}{2 \Gamma\left(1+\frac{N}{2}\right)}\right)^{\frac{1}{N}},
$$

where meas $(\Omega)$ denotes the Lebesgue measure of the set $\Omega$.

Moreover, let

$$
\mathrm{D}:=\sup _{x \in \Omega} \operatorname{dist}(x, \partial \Omega) .
$$

Simple calculations show that there is $x_{0} \in \Omega$ such that $\mathrm{B}\left(x_{0}, \mathrm{D}\right) \subseteq \Omega$.

Finally, we set

$$
\mathrm{k}:=\frac{D \sqrt{2}}{2 \pi^{\frac{N}{4}}}\left(\frac{\Gamma\left(1+\frac{N}{2}\right)}{D^{N}-\left(\frac{D}{2}\right)^{N}}\right)^{\frac{1}{2}},
$$

and

$$
K_{1}:=\frac{2 \sqrt{2} c_{1}\left(2^{N}-1\right)}{D^{2}}, \quad K_{2}:=\frac{2^{\frac{q+2}{2}} c_{q}^{q}\left(2^{N}-1\right)}{q D^{2}} .
$$

\section{Conclusion}

Our main result is the following theorem.

eroehTm 3.1. Let f: $\Omega \times R \rightarrow R$ be a Caratheodory function such that $\left(h_{1}\right)$ holds. Assume that $\left(h_{2}\right) F(x, \xi) \geq 0$ for every $(\mathrm{x}, \xi) \in \Omega \times R^{+}$;

$\left(h_{3}\right)$ there exist two positive constants $\mathrm{b}$ and $\mathrm{s}<2$ such that

$$
\mathrm{F}(\mathrm{x}, \xi) \leq b\left(1+|\xi|^{s}\right)
$$

for almost every $\mathrm{x} \in \Omega$ and for every $\xi \in R$;

$\left(h_{4}\right)$ there exist two positive constants $\gamma$ and $\delta$, with $\delta>\gamma k$ such that

$$
\frac{\inf f_{x \in \Omega} F(x, \delta)}{E \delta^{2}}<a_{1} \frac{K_{1}}{\gamma}+a_{2} K_{2} \gamma^{q-2},
$$

where $a_{1}, a_{2}$ are given in $\left(h_{1}\right)$ and $\mathrm{k}, K_{1}, K_{2}$ are given by (2.8) and (2.9).

Then, for each parameter $\lambda$ belonging to 


$$
\left.\Lambda_{(\gamma, \delta)}:=\right] \frac{2\left(2^{N}-1\right)}{D^{2}} \frac{\delta^{2} E}{\inf f_{x \in \Omega} F(x, \delta)}, \frac{2\left(2^{N}-1\right)}{D^{2}} \frac{1}{\left(a_{1} \frac{K_{1}}{\gamma}+a_{2} K_{2} \gamma^{q-2}\right)}
$$

the problem (1.1) possesses at least three weak solutions in $H_{0}^{1}(\Omega)$.

Proof: Let us apply theorem 2.1 with $\mathrm{X}=H_{0}^{1}(\Omega)$ and

$$
\phi(u):=\frac{\|u\|_{I}^{2}}{2}, \quad \psi(u):=\int_{\Omega} F(x, u(x)) d x,
$$

for every $\mathrm{u} \in X$. Let $\lambda>0$ and put

$$
J_{\lambda}(u):=\phi(u)-\lambda \psi(u), \quad \forall u \in X .
$$

As observed in section 2, $\phi: X \rightarrow R$ is a coercive, continuously Gâteaux differentiable and sequentially weakly lower semicontinuous functional whose Gâteaux derivative admits a continuous inverse on $X^{*}$. Moreover, $\psi$ is continuously Gâteaux differentiable with compact derivative and $\phi(0)=\psi(0)=0$.

Owing to $\left(h_{1}\right)$, one has that

$$
\mathrm{F}(\mathrm{x}, \xi) \leq a_{1}|\xi|+a_{2} \frac{|\xi|^{q}}{q}
$$

for every $(\mathrm{x}, \xi) \in \Omega \times R$.

Let $\mathrm{r} \in] 0,+\infty[$ and consider the function

Taking into account (3.1) it follows that

$$
\chi(r):=\frac{\sup _{\left.\left.u \in \phi^{-1}(]-\infty, r\right]\right)} \psi(u)}{r} .
$$

$$
\psi(u)=\int_{\Omega} F(x, u(x)) d x \leq a_{1}\|u\|_{L^{1}(\Omega)}+\frac{a_{2}}{q}\|u\|_{L^{q}(\Omega)}^{q} .
$$

Then, for every $\mathrm{u} \in X: \phi(u) \leq r$, due to (2.5), we get

Hence

$$
\psi(u) \leq\left(\sqrt{2 r} c_{1} a_{1}+\frac{2^{\frac{q}{2}} c_{q}^{q} a_{2}}{q} r^{\frac{q}{2}}\right)
$$

$$
\sup _{\left.\left.u \in \phi^{-1}(]-\infty, r\right]\right)} \psi(u) \leq\left(\sqrt{2 r} c_{1} a_{1}+\frac{2^{\frac{q}{2}} c_{q}^{q} a_{2}}{q} r^{\frac{q}{2}}\right) .
$$

Since, from (3.2), the following inequality holds

for every $r>0$.

$$
\chi(r) \leq\left(\sqrt{\frac{2}{r}} c_{1} a_{1}+\frac{2^{\frac{q}{2}} c_{q}^{q} a_{2}}{q} r^{\frac{q}{2}-1}\right)
$$

Next, put

$$
u_{\delta}(x):=\left\{\begin{array}{cc}
0 & \text { if } x \in \Omega-B\left(x_{0}, D\right), \\
\frac{2 \delta}{D}\left(D-\left|x-x_{0}\right|\right) & \text { if } x \in B\left(x_{0}, D\right)-B\left(x_{0}, \frac{D}{2}\right), \\
\delta & \text { if } x \in B\left(x_{0}, \frac{D}{2}\right) .
\end{array}\right.
$$

Clearly $u_{\delta} \in X$ and we have

$$
\phi\left(u_{\delta}\right)=\frac{1}{2}\left(\int_{\Omega}\left(\left|\nabla u_{\delta}(x)\right|^{2}+a(x)\left|u_{\delta}(x)\right|^{2}\right) d x\right.
$$




$$
\begin{gathered}
=\frac{1}{2}\left(\int_{B\left(x_{0}, D\right)-B\left(x_{0}, \frac{D}{2}\right)} \frac{(2 \delta)^{2}}{D^{2}} d x+\int_{B\left(x_{0}, D\right)-B\left(x_{0}, \frac{D}{2}\right)} a(x) \frac{(2 \delta)^{2}}{D^{2}}|D-| x-x_{0}||^{2} d x+\right. \\
\begin{array}{c}
\left.\int_{B\left(x_{0}, D\right)} a(x) \delta^{2} d x\right) \\
\quad \leq \frac{1}{2}\left(\frac{(2 \delta)^{2}}{D^{2}} \frac{\pi^{\frac{N}{2}}}{\Gamma\left(1+\frac{N}{2}\right)}\left(D^{N}-\left(\frac{D}{2}\right)^{N}\right)\right. \\
+\frac{(2 \delta)^{2}}{D^{2}} \sup _{x \in \Omega} \mathrm{a}(\mathrm{x}) \max _{x \in B\left(x_{0}, D\right)-B\left(x_{0}, \frac{D}{2}\right)}|D-| x-\left.x_{0}\right|^{2} \frac{\pi^{\frac{N}{2}}}{\Gamma\left(1+\frac{N}{2}\right)}\left(D^{N}-\left(\frac{D}{2}\right)^{N}\right) \\
\left.\quad+\delta^{2} \sup p_{x \in \Omega^{2}} \mathrm{a}(\mathrm{x}) \frac{\pi^{\frac{N}{2}}}{\Gamma\left(1+\frac{N}{2}\right)} \frac{D^{N}}{2^{N}}\right) \\
=\frac{1}{2} \frac{(2 \delta)^{2}}{D^{2}} \frac{\pi^{\frac{N}{2}}}{\Gamma\left(1+\frac{N}{2}\right)}\left(D^{N}-\left(\frac{D}{2}\right)^{N}\right)\left(1+M h+\frac{D^{2}}{2^{2}} M \frac{1}{2^{N}-1}\right) \\
=\frac{E}{2} \frac{(2 \delta)^{2}}{D^{2}} \frac{\pi^{\frac{N}{2}}}{\Gamma\left(1+\frac{N}{2}\right)}\left(D^{N}-\left(\frac{D}{2}\right)^{N}\right) .
\end{array}
\end{gathered}
$$

Bearing in mind that $\delta>\gamma k$, it follows that $\gamma^{2}<\phi\left(u_{\delta}\right)$.

At this point, by $\left(h_{2}\right)$ we infer

$$
\begin{aligned}
& \psi\left(u_{\delta}\right)=\int_{\Omega} F\left(x, u_{\delta}(x)\right) d x \geq \int_{B\left(x_{0}, \frac{D}{2}\right)} F(x, \delta) d x \geq \\
& i n f_{x \in \Omega} F(x, \delta) \frac{\pi^{\frac{N}{2}}}{\Gamma\left(1+\frac{N}{2}\right)} \frac{D^{N}}{2^{N}} .
\end{aligned}
$$

Hence, by (3.5) and (3.6) one has

$$
\frac{\psi\left(u_{\delta}\right)}{\phi\left(u_{\delta}\right)} \geq \frac{D^{2}}{2\left(2^{N}-1\right)} \frac{i n f_{x \in \Omega} F(x, \delta)}{\delta^{2} E}
$$

In view of (3.3) and taking into account $\left(h_{4}\right)$, we get

$$
\begin{aligned}
\chi\left(\gamma^{2}\right) & =\frac{\sup _{u \in \phi^{-1}(]-\infty, \gamma^{2} D} \psi(u)}{\gamma^{2}} \leq\left(\sqrt{2} \frac{c_{1}}{\gamma} a_{1}+\frac{2^{\frac{q}{2}} c_{q}^{q} a_{2}}{q} \gamma^{q-2}\right) \\
= & \frac{D^{2}}{2\left(2^{N}-1\right)}\left(a_{1} \frac{K_{1}}{\gamma}+a_{2} K_{2} \gamma^{q-2}\right)<\frac{D^{2}}{2\left(2^{N}-1\right)} \frac{\text { inf } f_{x \in \Omega} F(x, \delta)}{\delta^{2} E} \leq \frac{\psi\left(u_{\delta}\right)}{\phi\left(u_{\delta}\right)} .
\end{aligned}
$$

Therefore, the assumption $\left(a_{1}\right)$ of theorem 2.1 is satisfied.

Moreover, if $\mathrm{s}<2$, for every $\mathrm{u} \in X,|u|^{s} \in L^{\frac{2}{s}}(\Omega)$ and the Holder's inequality gives

Then, by (2.5), one has

$$
\int_{\Omega}|u(x)|^{s} d x \leq\|u\|_{L^{2}(\Omega)}^{s} \operatorname{meas}(\Omega)^{\frac{2-s}{2}}, \quad \forall u \in X .
$$

$$
\int_{\Omega}|u(x)|^{s} d x \leq c_{2}^{s}\|u\|^{s} \operatorname{meas}(\Omega)^{\frac{2-s}{2}}, \quad \forall u \in X .
$$

From (3.8) and due to condition $\left(h_{3}\right)$, it follows that

$$
J_{\lambda}(u) \geq \frac{\|u\|_{I}^{2}}{2}-\lambda \operatorname{bmeas}(\Omega)^{\frac{2-s}{2}}\|u\|^{s}-\lambda b \operatorname{meas}(\Omega), \quad \forall u \in X .
$$

Therefore, $J_{\lambda}$ is a coercive functional for every positive parameter, in particular, for every 


$$
\left.\lambda \in \Lambda_{(\gamma, \delta)} \subseteq\right] \frac{\phi\left(u_{\delta}\right)}{\psi\left(u_{\delta}\right)}, \frac{\gamma^{2}}{\sup _{\phi(u) \leq \gamma^{2}}}[.
$$

Then, also condition $\left(a_{2}\right)$ holds. Hence, all the assumptions of theorem 2.1 are satisfied, so that, for each $\lambda \in \Lambda_{(\gamma, \delta)}$ the functional $J_{\lambda}$ has at least three distinct critical points that are weak solutions of the problem (1.1).

Example 3.1 Let $\Omega$ be an open ball of radius one in $\left.R^{4}, \mathrm{q}:=3 \in\right] 2,4\left[\right.$ and $\mathrm{s}:=\frac{3}{2}<2$.

Pick $\mathrm{r}:=200$ and consider the function $\mathrm{f}: \mathrm{R} \rightarrow R$ defined by

$\mathrm{f}(\mathrm{t}):=\left\{\begin{aligned} 1+t^{2} & \text { if } t \leq 200 \\ 1+2000 \sqrt{2 t} & \text { if } t>200 .\end{aligned}\right.$

and $\mathrm{a}(\mathrm{x})=\frac{1}{e^{2} x^{\frac{1}{3}}}$.

Then, by theorem 3.1 , for each

$\lambda \in] \frac{18000 E}{40003}, \frac{12^{\frac{1}{4}}}{1+2 \sqrt{3} \pi^{2}} 4 \pi[$,

the problem(1.1) possesses at least three weak positive solutions in $H_{0}^{1}(\Omega)$.

\section{Acknowledgments}

The authors would like to thank the refere for the careful reading of this paper.

\section{References}

[1] D. Averna and G. Bonanno, A three critical points theorem and its applications to ordinary Dirichlet problems, Topol. Methods Nonlinear Anal. 22 (2003), 93-103.

[2] G.A. Afrouzi, S. Heidarkhani, Three solutions for a Dirichlet boundary value problem involving the p-Laplacian, Nonlinear. Anal. 66 (2007) 2281-2288.

[3] G. Bonanno, Some remarks on a three critical points theorem, Nonlinear Anal. 54 (2003), 651-665.

[4] G. Bonanno, G.M. Bisci, Three weak solutions for elliptic Dirichlet problems, J. Math. Anal. Appl. 382 (2011) 1-8.

[5] B. Ricceri, On a three critical points theorem, Arch. Math. (Basel) 75 (2000) 220-226.

[6] B. Ricceri, A general variational principle and some of its applications, J. Comput. Appl. Math. 113 (2000) 401-410.

[7] B. Ricceri, Existence of three solutions for a class of elliptic eigenvalue problems, Math. Comput. Modelling 32 (2000) 1485-1494. 